\title{
Surgical outcomes of and suggestions for carotid endarterectomy in patients on hemodialysis: report of 7 cases and literature review
}

\author{
Masaaki Hokari*, Daisuke Shimbo, Masayuki Gekka, Kazuki Uchida, Katsuyuki Asaoka and Koji Itamoto \\ Department of Neurosurgery, Teine Keijinkai Hospital, Japan
}

\begin{abstract}
Background: Carotid endarterectomy is an established surgical treatment for patients with severe carotid artery stenosis, but there are only a few reports dealing with the procedure in hemodialysis patients. We report the results of carotid endarterectomy in those on hemodialysis in our hospital.

Methods: We retrospectively analyzed the records of 7 hemodialysis patients who underwent carotid endarterectomy in our hospital from January 2014 to October 2019. During this period, 89 patients (including the 7 hemodialysis patients) underwent carotid endarterectomy. The characteristics and surgical outcomes in hemodialysis patients were compared with non-hemodialysis patients.

Results: The existence of severe plaque burden was significantly higher in hemodialysis patients than in non-hemodialysis patients $(86 \%$ vs. $27 \%$; $\mathrm{p}=0.001)$. The incidence of ischemic heart disease and arteriosclerosis obliterans and the percentage of the patients on dual anti-platelet therapy were significantly higher in the hemodialysis group than in the non-hemodialysis group. There was only one minor surgical complication in the hemodialysis patients (transient hoarseness). Although there was no perioperative mortality, three patients died during the follow-up period. Those that died were over 70 years old and the duration of their hemodialysis treatment was more than five years.
\end{abstract}

Conclusions: Our experience shows that while carotid endarterectomy can be safely performed in hemodialysis patients in the short term, asymptomatic patients who are over 70 years old with more than 5 years duration of hemodialysis should be basically offered optimal medical therapy, considering their poor long-term prognosis.

\section{Introduction}

The number of patients on dialysis in Japan currently exceeds 300,000 . Chronic dialysis is considered a risk factor for atherosclerosis [1]. For those on hemodialysis (HD), the relative risk of stroke and cerebral infarction is 5.2 and 2.0, respectively [2]. Furthermore, several authors have reported a higher incidence of carotid artery plaque in patients on HD [3,4]. According to a report by Pascazio, et al. $74 \%$ of HD patients have evidence of carotid plaque [4]. Maeda, et al. reported that carotid plaque was detected by ultrasonography in $87 \%$ of Japanese HD patients [3].

Carotid endarterectomy (CEA) is an evidence-based surgical treatment for severe carotid stenosis supported by high-profile randomized controlled trials [5-7]. However, many reports suggest high complication rates in patients with renal insufficiency [8-16]. There has been no consensus on the indications for CEA in HD patients, and a few large database studies have demonstrated a poor outcome of CEA in these patients [10,17]. However, due to the nature of these studies, they were lacking in detail. On the other hand, some individual institutional reports have made the argument that patients undergoing $\mathrm{HD}$ are at no greater risk for periprocedural complications after CEA [18-20]. However, these contradicting studies did not discuss perioperative management in detail. Therefore, the appropriate perioperative management of CEA in HD patients remains unclear.

Based on this background information, we present seven HD patients who underwent CEA and thoroughly review these cases with an emphasis on the perioperative management phase developed into CTEPH throughout seventeen years.

\section{Patients and methods}

\section{Patients}

The study was approved by the Institutional Review Board, with the need for informed consent waived because of the retrospective nature of this study. Comprehensive consent for using samples from medical examination for medical investigation was obtained. We retrospectively analyzed the records of $7 \mathrm{HD}$ patients who underwent CEA in our hospital from January 2014 to December 2019. During this period, 89 patients (including 7 HD patients) underwent CEA. An additional 65 patients were treated with carotid artery stenting (CAS). Of those treated with CAS, there was one patient on HD who had needed stenting after an artery treated with CEA restenosed.

\section{Clinical characteristics}

Clinical variables and outcomes of $7 \mathrm{HD}$ patients and 82 non-HD patients were compared. Clinical data were collected from the patients' medical records. The following factors were compared: age, gender,

${ }^{\star}$ Correspondence to: Hokari M, MD, PhD, Department of Neurosurgery, Teine Keijinkai Hospital, Maeda 1-12-1-40, Teine-ku, Sapporo, Hokkaido 006-0811, Japan, Tel: +81-11-811-6811; Fax: +81-11-685-2196; E-mail: masakari21@hotmail.com

Key words: carotid endarterectomy, hemodialysis, surgical outcomes, prognosis

Received: January 29, 2021; Accepted: February 05, 2021; Published: February 08, 2021 
current smoking, stenosis degree, plaque characteristics, contralateral lesion, hypertension (HT) (systolic blood pressure $>140 \mathrm{~mm} \mathrm{Hg}$ or diastolic blood pressure $>90 \mathrm{~mm} \mathrm{Hg}$ ) or current treatment status, diabetes mellitus (DM) (hemoglobin $A_{1 C}>6.5$ ) or current treatment status, hyperlipidemia (HL) (serum low-density lipoprotein cholesterol $>140 \mathrm{mg} / \mathrm{dl}$ ) or current treatment status, ischemic heart disease (IHD) or current treatment status, and previous treatment of arteriosclerosis obliterans (ASO) or diagnosed as femoral artery stenosis more than $50 \%$. All patients underwent preoperative screening for IHD to assess for risk of perioperative cardiac complications. We then consulted the cardiology department and, if required, performed coronary CT angiography or digital subtraction angiography. Regardless of the presence or absence of symptoms of angina pectoris, patients with severe stenosis of the coronary artery were regarded as having IHD.

The degree of carotid stenosis was determined according to the North American Symptomatic Carotid Endarterectomy Trial criteria (21), and estimated using 3-dimensional CT angiography (3D-CTA) or digital subtraction angiography (DSA). Contralateral stenosis (CCS) was defined as a presence of a more than $50 \%$ stenosis in the contralateral internal carotid artery.

Magnetic resonance (MR) imaging and/or carotid ultrasonography was performed for the evaluation of plaque characteristics. Black blood MRI was used with a fat-suppressed T1-weighted fast spin-echo sequence. The MR signal intensity of the carotid plaque in the area with highest rate of stenosis was classified as low or high compared with the intensity of the ipsilateral sternocleidomastoid muscle. For ultrasonography, carotid plaque was defined as an arterial wall lesion that projected into the vessel lumen. The plaque was qualitatively assessed as being predominantly ( $>50 \%$ area of plaque images) low (blood-like echogenicity), intermediate, or high (intensely bright echogenicity). Fragile plaque was defined as having a predominantly high signal by T1-weighted sequence and/or low echogenicity by ultrasonography. Heavy circumferential plaque calcification was defined as that which involved more than $75 \%$ of the vessel circumference at its narrowest portion by CTA, and entire circumferential calcification was defined as calcification of $100 \%$ of the vessel circumference at its narrowest portion.

Preoperative cerebral hemodynamics were evaluated using 123I-IMP single photon emission tomography (SPECT), with hypoperfusion defined as less than $80 \%$ compared to the contralateral hemisphere.

\section{CEA procedures}

All CEAs were performed under general anesthesia. Carotid shunts with intraoperative monitors of motor evoked potential were used as a matter of routine. CEA procedure was performed in our standard way as described previously [21]. Briefly, the carotid bifurcation was dissected, and then an intravenous bolus of heparin (3,000 units) was administered before carotid clamping. For precise and delicate plaque dissection, we routinely inserted internal shunt tubes. The superior thyroid and external carotid arteries were occluded with aneurysmal clips. The common and internal carotid arteries were occluded with vascular clamps. Arteriotomy was subsequently performed, and a 3-way internal shunt tube (Furui 3.0 or 3.5) was inserted in all patients. The duration of carotid clamping was 3-10 minutes. Blood pressure was maintained within the normal range during carotid clamping. The plaque was carefully dissected and removed under a surgical microscope. The arteriotomy was closed with a running suture, and the internal shunt tube was removed immediately before the arteriotomy closure was completed. After the successful completion of this task, the heparin was reversed. No patients underwent patch closure.

Additionally, the following were performed for all HD patients: 1) considering a high risk of postoperative neck hematoma in the patients with renal insufficiency [22], we used fibrin glue spray in order to reduce the risk of blood leak at the suture site; 2) because HD patients have high incidence of severe calcified plaque $[3,4]$ and carotid calcification often located mainly outside the carotid plaque [23], we took special care in performing the dissection between plaque and media. Furthermore, severe adhesive calcified plaque and media calcification were sometimes left in the media, rather than completely removed with media at the discretion of the surgical operator.

\section{Perioperative Management}

The patients with ipsilateral hemisphere hypoperfusion and, therefore, high risk of post-operative hyperperfusion syndrome were treated with strict blood pressure control and kept under general anesthesia for 2-5 days in the intensive care unit (ICU). Actually, 9 non-HD patients were treated in that manner, and the remaining 73 cases were carefully observed without sedation in the recovery room. One HD patient was considered high risk for hyperperfusion and treated under general anesthesia in the ICU. Because HD patients have generally high risk of perioperative systemic complications [10,17], the remaining $6 \mathrm{HD}$ patients were also transferred to ICU just after operation and carefully observed, but not under general anesthesia, for 3 days. HD was performed 1 day before and after surgery, using gabexate mesilate (FOY), in the place of heparin. HD was not performed on the day of surgery.

\section{Statistical Analysis}

All data were expressed as mean $\pm \mathrm{SD}$. The study variables were compared by $\chi^{2}$ test or unpaired t-test, as appropriate (Ekuseru-Toukei 2015; Social Survey Research Information Co., Ltd., Tokyo, Japan). P value of $<0.05$ was considered statistically significant.

\section{Results}

\section{Characteristics of the Patients}

The characteristics of the study population are presented in Table 1. The mean age was $71.1 \pm 14.2$ years for $H D$ patients and $72.7 \pm 6.3$ for non-HD patients. There was no significant difference in age between the two groups. The mean stenosis degree of HD patients was slightly higher than that of non-HD patients, but this was not significant $(84.3 \pm 12.4 \%$ for HD patients and $75.0 \pm 14.0 \%$ for non-HD patients, respectively). The existence of severe plaque was significantly higher in HD patients than in non-HD patients ( $86 \%$ vs. $27 \%$, $\mathrm{p}=0.001)$, but the incidence of fragile plaque in HD patients was significantly lower than in non-HD patients $(14 \%$ vs. $83 \%, \mathrm{p}<0.001)$. HD patients had a tendency for a higher incidence of contralateral lesions compared with the non-HD patients, but the difference was not significant. The incidence of IHD and ASO and the percentage of the patients on dual anti-platelet therapy (DAPT) was significantly higher in the HD group than in the non-HD group ( $71 \%$ vs. $34 \%$, $\mathrm{p}=0.049 ; 57 \%$ vs. $16 \%$, $\mathrm{p}=0.008$; and $43 \%$ vs. $13 \%, \mathrm{p}=0.04$, respectively).

Surgical complications were noted in 8 of a total 89 patients $(8.9 \%)$ : transient hoarseness in 2 , neck hematoma in 1 , transient cerebral ischemia in 1 , cerebral infarction in 2 (transient motor weakness due to ipsilateral lacunar infarction in 1 , and permanent mild hemiparesis due to contra-lateral cardiac embolic infarction in 1), and permanent hoarseness and dysphagia in 2 . These three patients' mRS deteriorated 
Table 1. characteristics of the study population

\begin{tabular}{|l|c|c|c|c|}
\hline \multicolumn{1}{|c|}{ Total } & HD & non-HD & P \\
\hline Age & 89 & 7 & 82 & \\
\hline Male & $72.6 \pm 7.1$ & $71.1 \pm 14.2$ & $72.7 \pm 6.3$ & 0.54 \\
\hline Syptomatic & $79(89 \%)$ & $5(72 \%)$ & $74(90 \%)$ & 0.13 \\
\hline Stenosis Degree & $43(48 \%)$ & $3(43 \%)$ & $40(49 \%)$ & 0.76 \\
\hline Entire caltification & $75.8 \pm 14.0 \%$ & $84.3 \pm 12.4 \%$ & $75.0 \pm 14.0 \%$ & 0.09 \\
\hline Severe caltification & $9(10 \%)$ & $4(57 \%)$ & $5(6 \%)$ & $*<0.001$ \\
\hline Fragile plaque & $28(31 \%)$ & $6(86 \%)$ & $22(27 \%)$ & $* 0.001$ \\
\hline contra-laterallesion & $69(78 \%)$ & $1(14 \%)$ & $68(83 \%)$ & $*<0.001$ \\
\hline DAPT & $18(20 \%)$ & $3(43 \%)$ & $15(18 \%)$ & 0.12 \\
\hline Anti-coagulant & $14(16 \%)$ & $3(43 \%)$ & $11(13 \%)$ & $* 0.04$ \\
\hline HT & $18(20 \%)$ & $2(29 \%)$ & $16(20 \%)$ & 0.57 \\
\hline DM & $70(79 \%)$ & $6(86 \%)$ & $64(78 \%)$ & 0.63 \\
\hline HL & $39(44 \%)$ & $3(43 \%)$ & $36(44 \%)$ & 0.96 \\
\hline IHD & $47(53 \%)$ & $2(29 \%)$ & $45(55 \%)$ & 0.18 \\
\hline Smoking & $33(37 \%)$ & $5(71 \%)$ & $28(34 \%)$ & $* 0.049$ \\
\hline ASO & $27(30 \%)$ & $2(29 \%)$ & $25(31 \%)$ & 0.92 \\
\hline Total surgical complication & $17(19 \%)$ & $4(57 \%)$ & $13(16 \%)$ & $* 0.008$ \\
\hline Mortality & $8(9.0 \%)$ & $1(14 \%)$ & $7(8.5 \%)$ & 0.61 \\
\hline Morbidity & $0(0 \%)$ & $0(0 \%)$ & $0(0 \%)$ & N/A \\
\hline Re-stenosis & $3(3.4 \%)$ & $0(0 \%)$ & $3(3.7 \%)$ & 0.61 \\
\hline & $6(6.7 \%)$ & $1(14 \%)$ & $5(6.1 \%)$ & 0.4 \\
\hline
\end{tabular}

HT: Hypertension; DM: Diabetes mellitus, HL: Hyperlipidemia; IHD: Ischemic heart disease; ASO: Arterio-sclerosis obliterans

only one point (from 1 to 2 or from 0 to 1 ). No patients deteriorated mRS more than 2 (No mortality and Morbidity). There was only one surgical complication in the HD patients (transient hoarseness), whose mRS deteriorated only one point (from 1 to 2 ). There were no mortality and no Morbidity in the HD patients. Restenosis after CEA occurred in 1 of $7 \mathrm{HD}$ patients (14.3\%) and in 5 of 82 non-HD patients $(6.1 \%)$.

Table 2 shows 7 cases of HD in detail. The causes of HD were diabetic nephropathy in 3 , hypertensive nephrosclerosis in 2 , and chronic glomerulonephritis (IgA nephropathy) in 2. Although there were no major cardiovascular adverse events, such as myocardial infarction, three perioperative minor cardiac events occurred ( 2 of AV block and 1 of angina), all of which occurred within 48 hours after surgery. Case 1 suffered from hypotension and Mobitz type 2 AV block 8 hours after surgery and was treated by catecholamine infusion. Case 4 suffered AV block during the first HD 26 hours after CEA and was treated by atropine injection. Due to prolonged bradycardia, after 1 week, a permanent pacemaker was implanted. Case 6 suffered from angina four hours after CEA, and, because abnormal ST elevation was noted on electrocardiogram, underwent emergent cardiac angiography. Fortunately, all of these cases eventually suffered no adverse cardiac mortality or morbidity.

Although there was no perioperative mortality, three patients (Case 1,3 , and 5) died during follow-up periods: 1 by multi-organ failure after head injury, 1 by electrolyte abnormality, and 1 by drowning in the bathtub. Case 1 had been an HD patient for 27 years when CEA was performed, Case 3 for 12 years, and Case 5 for 8 years. Thus, a long duration of HD therapy was a common characteristic in the patients that died.

\section{Representative Case (Case 4) Presentation}

A 84-year-old man with a 9-year history of hemodialysis for chronic renal failure due to diabetic nephropathy presented with mild right hemiparesis. MRI showed multiple acute infarctions in the left hemisphere (Figure 1A). CTA and echo demonstrated a severe stenosis of left cervical internal carotid artery with severe entire circumferential calcification (Figure 1B). He also had a history of HT, HL, DM, IHD, abdominal aortic aneurysm, and ASO, and he was treated with warfarin and DAPT. DAPT was continued and warfarin was replaced by heparin bridging therapy during the perioperative period. Left CEA was performed 3 weeks after cerebral infarction.

During the surgery, when we tried to clamp the carotid artery to insert a shunt tube, we could not completely stop the bleeding by using a single bulldog clamp due to heavy calcification; we needed three bulldog clamps to stop the bleeding. Additionally, severe adhesive calcified plaque and media calcification was left in the media, rather than completely removed with media.

After the surgery, he had no new neurological deficits and was transferred to ICU and carefully observed, but not under prolonged general anesthesia. Postoperative MRI revealed no high signals (Figure 1C), and MRA showed improvement of flow signal of right ICA (Figure 1D). However, on the next day, during his first postoperative HD in ICU (26 hours after CEA), complete Mobitz type 2 AV block was detected and immediately treated by atropine injection. After his bradycardia did not improve after 1 week, it was determined that he required permanent pacing. Although this cardiac incident occurred, he was discharged without any new neurological deficits and we confirmed he had been doing well for four years.

Table 2. 7 cases of HD in detail

\begin{tabular}{|l|l|c|c|c|c|c|c|}
\hline Case & Symptom & $\begin{array}{c}\text { Stenosis } \\
\text { degree }\end{array}$ & Site & HT & DM & HL & IHD \\
\hline $71 \mathrm{~F}$ & No (progression of stenosis) & 80 & Right & N & N & N & Y \\
\hline $74 \mathrm{M}$ & Left parietal infarction & 95 & Left & Y & N & N & Y \\
\hline $77 \mathrm{~F}$ & No (progression of stenosis) & 80 & Left & Y & N & N & N \\
\hline $84 \mathrm{M}$ & left front-parietal infarction & 80 & Left & Y & Y & Y & Y \\
\hline $75 \mathrm{M}$ & left frontal infaction & 90 & Left & Y & N & Y & N \\
\hline $76 \mathrm{M}$ & No (progression of stenosis) & 95 & Left & Y & Y & N & Y \\
\hline $40 \mathrm{M}$ & No (progression of stenosis) & 60 & Left & Y & Y & N & Y \\
\hline
\end{tabular}

WF: Warfarin; AS: Aspirin; Cil: Cilostazol; Clo: Clopidogrel
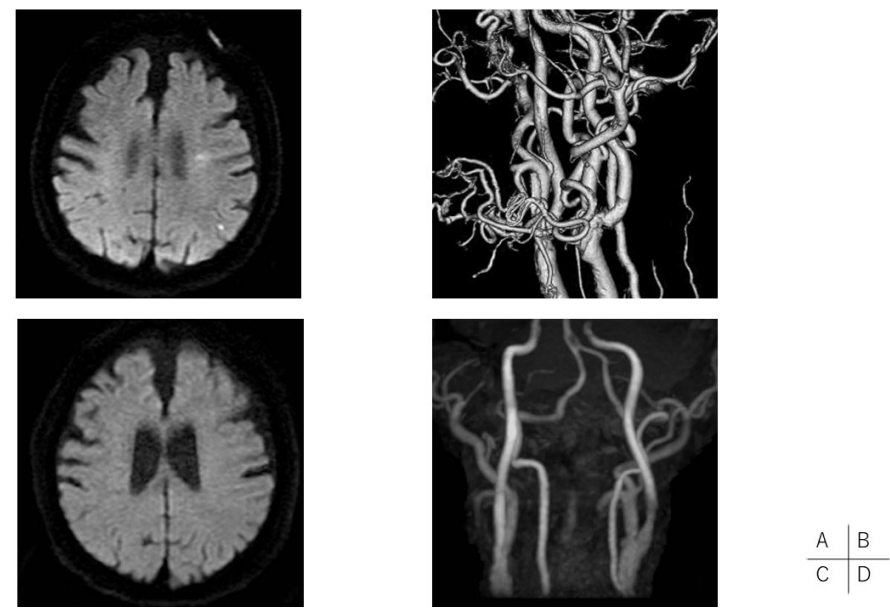

Figure 1. Pre- and post-operative neuroradiological findings of Case 4

Diffusion-weighted image (DWI) of magnetic resonance imaging (MRI) revealed several spotty high signals on the left cerebral hemisphere (Figure1A) and3-dimensional CT angiography demonstrated severe stenosis of left cervical internal carotid artery with severe entire circumferential calcification (Figure 1B). Postoperative DWI of MRI revealed no high signals (Figure 1C) and MRA showed improvement of flow signal of right ICA (Figure 1D). 


\section{Discussion}

Our study showed that HD patients have a tendency for a high incidence of contralateral lesions and severe stenosis degree compared with non-HD patients, and the incidence of severe plaque, IHD, ASO and the percentage of the patients on DAPT were significantly higher in the HD group than in the non-HD group. Due to methods in the CEA procedure for dealing with heavy circumferential plaque calcification and careful perioperative management in the ICU for those with a high risk of systemic disease, we could safely perform CEA for HD patients in the perioperative short term. However, taking into consideration a poor middle- or long-term prognosis, most of these patients with asymptomatic carotid stenosis should be offered optimal medical therapy, except for patients with a good long-term life expectancy.

\section{Indications for CEA in HD patients}

There have been several reports discussing risks and benefits of CEA for patients with renal insufficiency [8-16]. However, there has been no consensus on perioperative risks of CEA for patients with renal insufficiency, mostly because published studies did not use a universal definition of renal insufficiency [22]. As for HD patients, a few reports have discussed perioperative risk of CEA [10,18-20]. Although some investigators have published single-center reports that patients undergoing HD were at no greater risk for periprocedural complications when undergoing CEA with careful operative technique and management [18-20], Cooper, et al. showed that, through a large data-based study, outcomes were relatively poor and that survival was limited in the long term [10]. Although perioperative outcomes for asymptomatic patients were acceptable in their study, the longterm outcomes were very poor, with a less than $50 \% 3$-year survival in both asymptomatic and symptomatic patients. Therefore, they recommended optimizing medical management and avoiding CEA in asymptomatic patients, and selecting only symptomatic patients at very high risk for a second ischemic event for CEA. Per the Society for Vascular Surgery guidelines [23], asymptomatic patients with less than a 3-year life expectancy should receive medical management as the first-line therapy. This was expanded upon by AbuRahma, et al. who stated that CEA for symptomatic carotid stenosis can be justified only for carefully selected HD patients with acceptable operative risk and a good long-term life expectancy, and that asymptomatic patients should be offered best medical therapy [24].

Nevertheless, we assume high risk of stroke in HD patients when managed conservatively. Toyoda, et al. reported that cerebral infarction occurred more frequently during or less than 30 minutes after dialysis, especially in patients with large artery atherosclerosis [25]. Kashiwazaki, et al. reported the case of a patient with internal carotid artery occlusion who experienced frequent transient ischemic attacks during $\mathrm{HD}$, and they showed a marked deterioration in the cerebral oxygenation state in the ipsilateral hemisphere as a result of hypotension during HD, using near-infrared spectroscopy [26]. Based on these findings, the relative risk of cerebral infarction in patients with severe carotid stenosis is expected to be much higher in HD than non-HD patients. Therefore, we support the assertion that CEA can be performed in asymptomatic patients with severe or progressive carotid stenosis with a good long-term life expectancy, considering the high risk of stroke when managed conservatively.

Okawa, et al. examined 15 CEAs of $12 \mathrm{HD}$ patients and stated in their single center report that patients undergoing HD were at no greater risk for periprocedural complications when undergoing CEA with careful operative technique and management [20]. They suggested that CEA might be safe and effective for stroke prevention for HD patients, even in those who are asymptomatic. In their cases, the duration of $\mathrm{HD}$ in $10 \mathrm{CEAs}$ for asymptomatic lesions was all less than 10 years. Four cases were over 70 years old, but their duration of HD was less than 5 years. Our three cases who died within 2 years after CEA were all over 70 years old and their duration of HD was more than 5 years. Based on these findings, we think that asymptomatic patients who are over 70 years old with more than 5 years duration of HD should be offered best medical therapy.

Although CAS is an alternative modality for the treatment of carotid artery stenosis, HD patients have a high incidence of severely-calcified plaque [27] which is generally considered a relative contraindication for CAS because it prevents adequate stent expansion [28-30]. Applying this concept to our study, we think severe circumferential calcified plaque like that found in Case 4 cannot treated by CAS.

\section{Practical Devices of CEA for Carotid Plaque Characteristics in HD Patients}

Maeda, et al. reported that HD patients have a significantly higher incidence of carotid plaque and about $80 \%$ of them were calcified [3]. Most of our patients had severe plaque calcification, with more than half having entire circumferential calcification. Yamada, et al. analyzed carotid plaque calcification by $3 \mathrm{D}-\mathrm{CTA}$ and by histological examination of specimens resected by CEA [31]. They found that carotid calcification was most often located outside the carotid plaque and sometimes located further outside the internal elastic lamina, which was defined as arterial medial calcification. Therefore, the following potential problems in the CEA procedure could be considered in HD patients: 1) difficulty in clamping the carotid artery due to severe circumferential calcification and 2) risk of perforation during the plaque removal process due to adhesive calcified plaque and/or media calcification.

1) As shown in case 4, severe circumferential calcification could sometimes make it difficult to clamp the carotid artery. For such situations, therefore, we think it is necessary to prepare several spare bulldog clamps and enough exposure to deploy the clamps, especially the far to proximal common carotid artery area, thereby making it easier to manage the case.

2) Excessively careful dissection is needed to lessen the chance of perforation during the plaque removal process in CEA. Moreover, we believe that severe adhesive calcified plaque and/or media calcification should be left in the media, rather than completely removed. Murahashi, et al. detail their surgical recommendations for CEA in HD patients in their Japanese language article [19]. They also emphasized that careful dissection was essential and adhesive calcified plaque should be left in the media. We used a dissector with a semi-sharp side (KamiyamaIwasaki's dissector), which was valuable in the identification of the border between the atheromatous plaque and media and the exfoliation of very tough, adhesive intima [32]. Yasuda, et al. reported that while dull dissection could exfoliate most of carotid plaque in the CEA procedure, sometimes sharp dissection was necessary for calcified plaque. However, it should be pointed out that sharp dissection raises the risk of perforation. Therefore, semi-sharp dissection by a semisharp spatula can prove to be really useful. By using this method, we fortunately did not experience any carotid perforations.

Several investigators have reported that risk factors for neck hematoma after CEA consisted of uncontrolled hypertension [33], combined platelet inhibition, use of dextran, non-reversal of heparin, 
and elevated creatinine [34]. Hamdan, et al. reported a higher rate of hematoma formation in patients with renal insufficiency [12]. In this study, the percentage of the patients on multiple antithrombotic drugs was higher in those on HD. Therefore, we should be extremely cautious in monitoring for postoperative bleeding. For example, to prevent neck bleeding after CEA, keeping the operative field dry and bloodfree during surgery is essential. We usually used fibrin glue in the case of oozing from a sutured carotid artery without a need for additional suturing. However, even if there was no oozing from the suture site, we employed a polyglycolic acid sheet soaked with fibrin glue to prevent bleeding from the sutured carotid artery for all HD patients. A previous study reported that fibrin sealant reduced suture line bleeding [35]. Perhaps the use of such procedural devices prevented neck hematoma formation after CEA in our HD patients.

\section{Appropriate perioperative Management of CEA in HD patients}

As in previous reports [10,17] we also found the incidence of systemic complications, such as HT, DM, HL, IHD, ASO, to be higher in HD patients. Therefore, we should be acutely aware of perioperative systemic complications, especially cardiac complications. Because of this reasoning, all HD patients were transferred to the ICU just after operation and carefully observed with close cardiac monitoring. In a past report, myocardial infarction after CEA often occurred within 24 hours [36]. Actually, 3 of 7 cases experienced minor cardiac incidents within 24 hours after CEA, but rapid detection and response in the ICU likely helped to avoid progression to major complications. Therefore, we think it reasonable to carefully observe HD patients in an ICU setting for several days postoperatively. Needless to say, early consultation with cardiologists, nephrologists, and anesthesiologists is vital. We believe that such interventions are factors that allowed us to avoid perioperative major systemic complications in the HD patients after CEA.

\section{Conclusions}

Our study showed that HD patients have a significantly high incidence of severe plaque, IHD, ASO, and percentage of DAPT use. Due to methods in the CEA procedure specifically tailored for heavy circumferential plaque calcification and careful postoperative management in the ICU, we could safely perform CEA for HD patients in the perioperative short term. However, considering poor middleor long-term prognosis, asymptomatic patients who are over 70 years old with more than 5 years duration of HD should be basically offered optimal medical therapy.

\section{Sources of Funding}

None.

\section{Declaration of Interest}

None.

\section{Acknowledgments}

The authors deeply thank Yui Suzuki for her technical assistance.

\section{References}

1. Iseki K, Fukiyama K (1996) Predictors of stroke in patients receiving chronic hemodialysis. Kidney Int 50: 1672-1675.

2. Iseki K, Kinjo K, Kimura Y, Osawa A, Fukiyama K (1993) Evidence for high risk of cerebral hemorrhage in chronic dialysis patients. Kidney Int 44: 1086-1090.
3. Maeda N, Sawayama Y, Tatsukawa M, Okada K, Furusyo N, et al. (2003) Carotid artery lesions and atherosclerotic risk factors in japanese hemodialysis patients. Atherosclerosis 169: 183-192.

4. Pascazio L, Bianco F, Giorgini A, Galli G, Curri G, et al. (1996) Echo color doppler imaging of carotid vessels in hemodialysis patients: Evidence of high levels of atherosclerotic lesions. Am J Kidney Dis 28: 713-720. [Crossref]

5. Group ECSTC (1991) Mrc european carotid surgery trial: Interim results for symptomatic patients with severe (70-99\%) or with mild (0-29\%) carotid stenosis. European carotid surgery trialists' collaborative group. Lancet 337: 1235-1243. [Crossref]

6. Study ECftACA (1995) Endarterectomy for asymptomatic carotid artery stenosis. JAMA 273: 1421-1428.

7. Barnett HJ, Taylor DW, Eliasziw M, Fox AJ, Ferguson GG, et al. (1998) Benefit of carotid endarterectomy in patients with symptomatic moderate or severe stenosis. North american symptomatic carotid endarterectomy trial collaborators. $N$ Engl J Med 339: 1415-1425. [Crossref]

8. Adil MM, Saeed F, Chaudhary SA, Malik A, Qureshi AI (2016) Comparative outcomes of carotid artery stent placement and carotid endarterectomy in patients with chronic kidney disease and end-stage renal disease. J Stroke Cerebrovasc Dis 25: 1721-1727.

9. Becker BN, Himmelfarb J, Henrich WL, Hakim RM (1997) Reassessing the cardiac risk profile in chronic hemodialysis patients: A hypothesis on the role of oxidant stress and other non-traditional cardiac risk factors. J Am Soc Nephrol 8: 475-486. [Crossref]

10. Cooper M, Arhuidese IJ, Obeid T, Hicks CW, Canner J, et al. (2016) Perioperative and long-term outcomes after carotid endarterectomy in hemodialysis patients. JAMA Surg 151: 947-952.

11. Debing E, Van den Brande P (2006) Chronic renal insufficiency and risk of early mortality in patients undergoing carotid endarterectomy. Ann Vasc Surg 20: 609-613.

12. Hamdan AD, Pomposelli FB Jr., Gibbons GW, Campbell DR, LoGerfo FW (1999) Renal insufficiency and altered postoperative risk in carotid endarterectomy. J Vasc Surg 29: 1006-1011. [Crossref]

13. London GM, Marchais SJ, Guerin AP, Metivier F (2002) Impairment of arterial function in chronic renal disease: Prognostic impact and therapeutic approach. Nephrol Dial Transplant 11: 13-15.

14. Plecha EJ, King TA, Pitluk HC, Rubin JR (1993) Risk assessment in patients undergoing carotid endarterectomy. Cardiovasc Surg 1: 30-32.

15. Protack CD, Bakken AM, Saad WE, Davies MG (2011) Influence of chronic renal insufficiency on outcomes following carotid revascularization. Arch Surg 146: 11351141. [Crossref]

16. Lammeren GWV, Moll FL, Blankestijn PJ, de Kleijn DP, Bots ML, et al. (2011) Decreased kidney function: An unrecognized and often untreated risk factor for secondary cardiovascular events after carotid surgery. Stroke 42: 307-312.

17. Yuo TH, Sidaoui J, Marone LK, Makaroun MS, Chaer RA (2015) Revascularization of asymptomatic carotid stenosis is not appropriate in patients on dialysis. J Vasc Surg 61: 670-674. [Crossref]

18. Amin A, Golarz S, Scanlan B, Hashemi H, Mukherjee D (2008) Patients requiring dialysis are not at risk of greater complication after carotid endarterectomy. Vascular 16: 167-170. [Crossref]

19. Murahashi T, Kamiyama K, Osato T, Watanabe T, Ogino T, Sugio H, et al. (2017) Treatment strategy and results of carotid endarterectomy in chronic renal failure patients. No Shinkei Geka 45: 127-132. [Crossref]

20. Okawa M, Ueba T, Ogata T, Abe H, Higashi T, et al. (2014) Long-term morbidity and mortality of carotid endarterectomy in patients with end-stage renal disease receiving hemodialysis. J Stroke Cerebrovasc Dis 23: 545-549.

21. Iwasaki M, Kuroda S, Nakayama N, Hokari M, Yasuda H, et al. (2011) Clinical characteristics and outcomes in carotid endarterectomy for internal carotid artery stenosis in a japanese population: 10-year microsurgical experience. J Stroke Cerebrovasc Dis 20: 55-61. [Crossref]

22. Kretz B, Abello N, Brenot R, Steinmetz E (2010) The impact of renal insufficiency on the outcome of carotid surgery is influenced by the definition used. $J$ Vasc Surg $51: 43-50$.

23. Ricotta JJ, Aburahma A, Ascher E, Eskandari M, Faries P, et al. (2011) Updated society for vascular surgery guidelines for management of extracranial carotid disease: Executive summary. J Vasc Surg 54: 832-836. 
24. AbuRahma AF, Mousa AY, Stone PA (2011) Shunting during carotid endarterectomy. $J$ Vasc Surg 54: 1502-1510.

25. Toyoda K, Fujii K, Fujimi S, Kumai Y, Tsuchimochi H, et al. (2005) Stroke in patients on maintenance hemodialysis: A 22-year single-center study. Am J Kidney Dis 45: $1058-1066$.

26. Kashiwazaki D, Kuroda S, Terasaka S, Iwasaki Y (2007) Detection of hemodynamic transient ischemic attack during hemodialysis with near-infrared monitoring in a patient with internal carotid artery occlusion. Surg Neurol 68: 292-294. [Crossref]

27. Coll B, Betriu A, Martinez-Alonso M, Amoedo ML, Arcidiacono MV, et al. (2011) Large artery calcification on dialysis patients is located in the intima and related to atherosclerosis. Clin J Am Soc Nephrol 6: 303-310. [Crossref]

28. Choi HM, Hobson RW, Goldstein J, Chakhtoura E, Lal BK, et al. (2004) Technical challenges in a program of carotid artery stenting. J Vasc Surg 40:746-751.

29. Katano H, Mase M, Nishikawa Y, Yamada K (2014) Surgical treatment for carotid stenoses with highly calcified plaques. J Stroke Cerebrovasc Dis 23: 148-154. [Crossref]

30. Roubin GS, Iyer S, Halkin A, Vitek J, Brennan C (2006) Realizing the potential of carotid artery stenting: Proposed paradigms for patient selection and procedural technique. Circulation 113: 2021-2030.
31. Yamada S, Oshima M, Watanabe Y, Ogata H, Hashimoto K, et al. (2014) Intramural location and size of arterial calcification are associated with stenosis at carotid bifurcation. Eur J Radiol 83: 957-963.

32. Yasuda H, Kuroda S, Nakayama N, Kamiyama H, Saito H, et al. (2008) Kamiyamaiwasaki dissector in microsurgery for cerebral aneurysm and internal carotid artery stenosis. Currently Practical Neurosurgery 18: 489-495.

33. Kunkel JM, Gomez ER, Spebar MJ, Delgado RJ, Jarstfer BS, et al. (1984) Wound hematomas after carotid endarterectomy. Am J Surg 148: 844-847. [Crossref]

34. Comerota AJ, Difiore R, Tzilinis A, Chahwan S (2012) Cervical hematoma following carotid endarterectomy is morbid and preventable: A 12-year case-controlled review. Vasc Endovascular Surg 46: 610-616. [Crossref]

35. Milne AA, Murphy WG, Reading SJ, Ruckley CV (1995) Fibrin sealant reduces suture line bleeding during carotid endarterectomy: A randomised trial. Eur J Vasc Endovasc Surg 10: 91-94. [Crossref]

36. Blackshear JL, Cutlip DE, Roubin GS, Hill MD, Leimgruber PP, et al. (2011) Myocardial infarction after carotid stenting and endarterectomy: Results from the carotid revascularization endarterectomy versus stenting trial. Circulation 123: 25712578. [Crossref]

Copyright: (C2021 Hokari M. This is an open-access article distributed under the terms of the Creative Commons Attribution License, which permits unrestricted use, distribution, and reproduction in any medium, provided the original author and source are credited. 